\title{
Novel Insights from Genetic and Molecular Characterization of the Human Clock
}

\author{
L.J. PTÁČEK, ${ }^{* \dagger}$ C.R. JONES, ${ }^{\ddagger}$ AND Y.-H. FU* \\ *Department of Neurology, University of California, San Francisco, California 94158-2324; \\ ${ }^{\dagger}$ Howard Hughes Medical Institute, University of California, San Francisco, California 94158-2324; \\ ${ }^{\ddagger}$ Department of Neurology, University of Utah, Salt Lake City, Utah 84132-2450
}

\begin{abstract}
Biological rhythms govern the ebb and flow of life on planet Earth. Animals have an internal timekeeping mechanism that precisely regulates 24-hour rhythms of body function and behavior and synchronizes them to the day/night cycle. Circadian pacemakers trigger behavioral and physiological processes that dictate our daily rhythms. Despite the importance of the circadian clock to all aspects of our physiology and behavior, the opportunity to probe the human circadian clock only recently became possible with the recognition of Mendelian circadian variants in people (familial advanced sleep phase syndrome, FASPS). We have now cloned several genes and identified mutations causing FASPS. Study of these genes and the proteins they encode and engineering of the human mutations into mouse models are allowing study of this fascinating phenotype and yielding novel insights into circadian regulation in humans. Ultimately, such work will allow us to understand the similarities and differences between the human clock and those of model organisms. In addition, recent studies have also linked disruption of the circadian clock with numerous ailments, including cancer, cardiovascular diseases, asthma, and learning disorders. Thus, studying the molecular mechanism of human circadian rhythmicity will have an enormous impact on our understanding of human health and disease. It should also lead to new strategies for pharmacological manipulation of the human clock to improve the treatment of jet lag, various clock-related sleep and psychiatric disorders, and other human diseases.
\end{abstract}

\section{HUMAN BEHAVIORAL GENETICS}

Studies of genetic behaviors in humans have been hampered by the complexity of behavioral phenotypes, in addition to locus and allelic heterogeneity that is certain to exist. The identification of a Mendelian circadian variant of humans (FASPS) was an exciting advance in the field, as the existence of such families suggested that cloning of a human behavioral gene was, in fact, a reasonable undertaking (Jones et al. 1999). The variants found in an extant human population have been consistent with life on our planet. In contrast, forward genetic screens that have been performed in other organisms have focused on mutants of large effect. Many of these would likely be selected against in evolution. Thus, in human FASPS, the a priori expectation is that period variants in humans would be smaller in amplitude than those identified in genetic screens.

In model organisms, most mutation screens have focused on measuring the circadian period and identifying alleles that lead to very short or very long $\tau$. Period measurements are used because they are relatively easy to perform and easily quantitated in organisms such as Drosophila and mice. However, measuring periods in humans is very challenging. Consequently, it would be very difficult to get periods on many family members from the same pedigree as well as from many other pedigrees. Thus, in the new field of human circadian genetics, the focus has been on phenotypes affecting phase (phase advance: "morning larks" or phase delay: "night owls"). Phase alterations can result from period alterations but, theoretically, could also be caused by altered phase angle of entrainment or coupling of clock to behavioral outputs. Theoretically, phase advance/delay may result from mutations that affect period and/or phase angle of entrainment.
FASPS was identified because of a very dramatic advance of the sleep phase that was recognized initially in a single individual. In contrast to the phase advance seen in the ASPS of aging, FASPS typically has its onset in childhood or young adult life and persists into old age, sometimes apparently worsening with aging (Jones et al. 1999). The phase was advanced 4-6 hours in FASPS subjects compared to controls, but sleep quality and quantity were normal. Subsequently, we have identified a large number of families segregating dominant FASPS alleles.

Identification of FASPS in a large cohort of FASPS families thus provided the opportunity to search for genes and variants that cause period alterations as well as phase angle of entrainment variants in the general population.

\section{LESSONS FROM GENETIC AND MOLECULAR DISSECTION OF CLOCKS IN THE MODEL ORGANISMS}

Extensive work in a number of model organisms has led to a model of the circadian molecular clock consisting of interlocked negative and positive transcriptional/translational feedback loops that oscillate with a period of approximately 24 hours. Not surprisingly, there are shared elements from the clocks of diverse organisms, from Neurospora to Drosophila and rodents. However, it is also not surprising that the molecular components that have been identified in mammals have elucidated a somewhat more complex clock mechanism, presumably with more precise regulation of more complex organisms. It is also interesting that there are differences between the clocks of invertebrates and vertebrates, and it is very likely that differences exist even between humans and rodents. Most 
rodents have circadian periods of less than 24 hours, whereas human periods tend to be slightly longer than 24 hours. The molecular basis of phase differences is not known; mice are nocturnal, whereas humans are diurnal organisms. Therefore, the ability to begin to probe the genetic basis of human clock function was an exciting and important advance, as it allows one to begin to understand not only the similarities, but also the differences that exist between clocks in humans and other organisms.

\section{Advanced Sleep Phase Syndrome and Aging}

The advanced sleep phase syndrome of aging (ASPS of aging) is defined as a tendency to awake and go to sleep earlier than desired as one gets older. It has recently been demonstrated that this tendency begins at about the age of 20 but tends to become of greater amplitude as individuals age (Roenneberg et al. 2004). Many people tend to go to sleep and awaken earlier as they age but are not troubled by this sleep phase advance. Although it is likely that this results from the same phenomenon causing ASPS of aging, clinicians do not refer to this as ASPS of aging because it is not troublesome to many subjects.

\section{Familial Advanced Sleep Phase Syndrome}

FASPS was first recognized in a large Utah pedigree, segregating an allele that led to a lifelong tendency to wake up and sleep at very early times (biological tendency to awaken spontaneously between 1:00 a.m. and 5:30 a.m. and to fall asleep spontaneously between 5:30 p.m. and 8:30 p.m.). To identify the underlying biological preference, it is necessary to dissect away the psychosocial and familial-cultural factors that impinge on the innate tendency. Furthermore, environmental factors such as artificial light, alcohol, and caffeine also modify expression of the preferred biological sleep/wake times.

The trait was recognized as early as 8 years of age in one subject, but frequently, the subjects to do not meet formal criteria until their mid twenties. This is likely because the delayed sleep phase syndrome (DSPS) of adolescence can in many cases mask the strong phase advance of FASPS. The trait segregates as a highly penetrant autosomal dominant trait (Jones et al. 1999). Affected individuals from this first pedigree were studied and had normal sleep quality and quantity. Melatonin and temperature rhythms, as well as sleep and wake times, were advanced by $4-6$ hours in affected subjects relative to controls. The proband from the family underwent a free-running period measurement and was shown to have a period nearly 1 hour shorter than age- and gendermatched controls (Jones et al. 1999).

Analysis of subjects and families included the HorneOstberg questionnaire, although it is now clear that the Munich Chronotype questionnaire (Zavada et al. 2005) may be better for assessing phase in individuals from FASPS families. In addition, subjects completed a general sleep-screening questionnaire to rule out sleep disorders such as obstructive sleep apnea, narcolepsy, or restless leg syndrome, which might lead to excessive daytime sleepiness and early sleep times. The Beck depres- sion inventory and neuropsychology interview were important to assess whether subjects had major depression, which could lead to early morning awakening. In contrast to the early morning awakening common in patients with depression, FASPS individuals wake up feeling energetic and refreshed. Finally, all subjects undergo a 1-hour structured interview that attempts to disentangle the many psychosocial, familial-cultural, and self-imposed decisions that humans tend to overlay on their underlying biologic sleep and wake tendencies.

\section{POSITIONAL CLONING OF THE FIRST HUMAN CIRCADIAN RHYTHM GENE}

\section{Period 2}

Genetic study of the Utah family described by Jones et al. led to the mapping of the FASPS allele to chromosome $2 q$ (Toh et al. 2001). At the time of the mapping, very little genomic sequence was available for chromosome $2 \mathrm{q}$, and thus, physical mapping of the 3-4-Mb region of chromosome $2 q$ was necessary and led to identification of more than $40 \mathrm{cDNAs}$ within the critical region. Sequencing of these candidate cDNAs identified one to be a homolog of the Drosophila period gene. Sequencing of this gene ( $h P E R 2)$ revealed a serine-to-glycine mutation at position 662 that segregated with affected members in the family (Toh et al. 2001). There was one small branch containing three "affected" individuals that did not carry the mutation. On the basis of in vitro functional studies and, ultimately, animal modeling of the human mutation, it was shown that these individuals represented FASPS phenocopies. Although these individuals had a strong morning lark tendency, they were the least advanced of any of the individuals who had been classified as affected in this family. These phenocopies underscore the challenges of behavioral genetics where strong morning lark phenotypes in individuals were not caused by the FASPS mutation segregating in this family. In addition, they emphasize the power of large Mendelian families for mapping and identifying genes despite the challenges of behavioral genetics.

This serine-to-glycine mutation led to hypophosphorylation of the PER2 polypeptide by casein kinase I (CKI). Coimmunoprecipitation experiments demonstrated that the region harboring this mutation was a point where CKI interacts with PER2. Sequence alignment of mammalian period proteins also showed that the mutant serine residue was the first of five that were separated from one another by two amino acids. This motif is consistent with a recognition site for CKI. Finally, replacement of the serine at position 662 with acidic residues was shown to reconstitute the gel-shift due to phosphorylation of downstream residues when the PER2 polypeptide was treated with recombinant-purified CKI (Toh et al. 2001).

\section{Casein Kinase $I \delta$}

With the increasing density of the human genome sequence across the genome and a growing collection of well-characterized FASPS subjects, it then became possible to sequence candidate gene exons to search for muta- 
tions in other FASPS families. The second mutation found was in a moderate-sized family with a strong FASPS allele in five individuals. An additional sixth individual was a 14-year-old boy at the time of his assessment, and although he was a strong morning lark for a teenager, he did not meet the strict clinical criteria of FASPS (Jones et al. 1999; Toh et al. 2001). All six of these individuals carried a missense mutation in the casein kinase I $\delta(\mathrm{CKI} \delta)$ gene in a highly conserved region of the protein. The mutation occurred six amino acids carboxyl terminal to the catalytic lysine (K38) of CKI $\delta$. This residue was completely conserved from the Drosophila double-time protein through human CKI. This mutation led to hypophosphorylation of phosvitin and $\alpha$-casein, as well as mammalian PER2 proteins (Xu et al. 2005). Generation of bacterial artificial chromosome (BAC) transgenic mice carrying the T44A mutation revealed a shortening of period of approximately 0.4 hours (Xu et al. 2005).

Phosphorylation of the region downstream from S662 by CKI thus represents a convergence of two different genes (CKI and Per2) where mutations cause the same FASPS phenotype. In the case of PER2, an inability to phosphorylate S662 (because it has mutated to a glycine) leads to hypophosphorylation, despite the presence of wild-type CKI. In the case of the $\tau$ mutant hamster and the human FASPS family with the CKI $\delta$ mutation, hypophosphorylation results from a mutant kinase (CK1E in the case of the $\tau$ mutant hamster and $\mathrm{CK} 1 \delta$ in a human FASPS family).

\section{IN VITRO BIOCHEMICAL EXPERIMENTS TO ASSESS PHOSPHORYLATION IN THE REGION DOWNSTREAM FROM PER2 S662}

Oligopeptides with the sequence including S662 and downstream residues were synthesized with or without a phosphate covalently linked to the serine corresponding to position 662 in PER2. Qualitative assays measuring incorporation of ${ }^{32} \mathrm{P}$ revealed that there was robust phosphorylation of the peptide with the covalently linked phosphate but not for the peptide without. Subsequently, a quantitative assay was used to demonstrate that approximately 4 moles of phosphate were added per mole of PER2 substrate with the covalently linked phosphate $(\mathrm{Xu}$ et al. 2007). Phosphopeptide-mapping experiments showed that the tyrosine and threonine residues in the oligopeptides were not phosphorylated, consistent with the prediction that serial serine phosphorylations occurred at every third amino acid downstream from position 662. This experiment also suggests that CKI does not phosphorylate S662 but, rather, that a priming event is required due to phosphorylation by another kinase before CK1 can phosphorylate the four downstream serines (Xu et al. 2007).

\section{PER2 MICE CARRYING THE HUMAN FASPS PER2 MUTATION RECAPITULATES AN FASPS PHENOTYPE}

Transgenesis was accomplished using a human BAC that contained the entire Per 2 gene with more than $50 \mathrm{~kb}$ of genomic sequence upstream of the ATG start site. The advantage of this strategy is that the genomic gene is large and, with intronic and intragenic genomic sequences, is typically buffered from the position effects that are so common with transgenesis using cDNAs. Furthermore, the cis-regulatory elements are frequently included in the upstream sequence, leading to expression patterns that are faithful to the endogenous genes.

A wild-type $h P E R 2$ transgenic was generated as a control for increased PER2 dosage and for the use of the human gene. Homologous recombination in bacteria was then used to generate the S662G mutation. Finally, a third transgene was generated encoding an aspartate at position 662 , to mimic a constitutively phosphorylated S662. Multiple lines of each of these transgenes were generated, and the period of wild-type transgenics carrying one to three copies of the hPer 2 transgene had a completely normal period. In contrast, the $\mathrm{S} 662 \mathrm{G}$ transgenic animals had a period that was approximately 2 hours shorter than controls, whereas the S662D transgenic animals had a period that was approximately one-half-hour longer than wildtype. When studied in a light/dark 12:12 paradigm, the S662G mice were phase-advanced and began running on the wheel 4-6 hours before the lights were turned off and decreasing activity hours before the lights came back on.

Further study of the cycling of various mRNAs was accomplished in liver and fibroblast extracts from these animals and showed that both the mouse and human Per2 mRNA was phase-advanced and decreased in levels in the S662G mice and phase-delayed and of higher amplitude in S662D mice when compared to wild-type controls (Xu et al. 2007). Contrary to the expectation, the S662G mutation did not appear to manifest its effects through decreased protein stability but through decreased transcription of the Per 2 gene. The mutant hPer 2 protein had a dominant effect on decreased transcription of both mPer2 and hPer2.

\section{CK1 $\delta$ DOSAGE EXPERIMENTS OF S662G AND S662D TRANSGENIC PER2 MICE}

Heterozygosity for a null mutation in CKI $\delta$ does not have an effect on period (Xu et al. 2005) (homozygous knockout is lethal). Transgenesis using a wild-type CKI $\delta$ BAC transgene with one to three copies per genome also does not have an effect on period. However, the CKI $\delta$ transgene, when crossed onto PER2 S662G transgenic mice led to a further shortening of period, whereas crossing a null allele for CKI $\delta$ onto the same transgenic animals led to a less short period than the Per 2 mutant alone. In addition, crossing one null CK $1 \delta$ allele onto the S662D Per2 transgenic mouse led to further prolongation of the period. Taken together, these data suggest a model whereby CKI is exerting opposite effects through phosphorylation of different sites. Phosphorylations downstream from residue 662 lead to increased transcription and increased PER2 protein, whereas phosphorylation at another site (presumably in PER2, although the possibility remains that this could be through an indirect effect) leads to increased PER2 degradation and lower PER2 levels. Changing CK1 $\delta$ dosage therefore has little effect when wild-type PER2 is present. However, with a muta- 
tion at position $662, \mathrm{CKI} \delta$ is unable to activate transcription and therefore PER2 levels are lower and the normal process of degradation continues, leading to decreased PER2 levels at all time points in the circadian day.

\section{FUTURE DIRECTIONS AND PERSPECTIVES}

Following on many discoveries using model organisms, tremendous insights into the workings of circadian clocks have been realized. During the last decade, these types of genetic experiments have now been extended into humans with recognition and characterization of a Mendelian circadian phenotype in humans and cloning of genes causing the FASPS phenotype. It is interesting that some elements of the circadian clock are conserved across very large evolutionary times, but certainly, there is evidence that differences also occur between the clocks of different species. Studying genes and mutations identified in humans with strong circadian phenotypes is focusing attention on interesting regions of these proteins and allowing biological experiments that will lead to greater insights not only into the workings of the circadian clock mechanism, but also its relationship to other metabolic and biochemical systems. Because the circadian clock regulates many biological processes, including (but not limited to) the immune system, cardiopulmonary systems, and cell cycle regulation, it is likely that knowledge of the workings of the human clock will come to bear on our understanding not only of health, but also of the relationship of the circadian clock and disease. Generation of human circadian mutations in other organisms and comparing phenotypes across different species are certain to provide interesting information regarding similarities and differences that the human clock shares with different organisms.

\section{APPLICATION OF KNOWLEDGE TO THE SPECTRUM OF CHRONOTYPES IN THE GENERAL HUMAN POPULATION}

With the identification of genes known to function in the circadian system and with the recognition of nonsynonymous genetic variants that exist in some of these genes, some groups have set out to look for associations of certain clock gene alleles with extreme chronotypes (morning larks or night owls). To date, a number of positive associations have been recognized (Katzenberg et al. 1998; Ebisawa et al. 2001; Robilliard et al. 2002; Archer et al. 2003; Takano et al. 2004; Carpen et al. 2005, 2006; Mishima et al. 2005). Although in each case, the actual effect of one allele and the strength of the effect in the association of that allele with either morningness or eveningness have been statistically significant, they also tend to be relatively small effects. Thus, it is likely that the broad spectrum of sleep time preferences in the population, separate from the Mendelian FASPS and FDSPS families, results from the combination of different alleles in a number of different circadian genes that add up to manifest in different tendencies in different individuals.

It is also likely that there are genetic contributions not only to circadian period, but to total sleep requirement, tendency to jet lag when crossing time zones, plasticity of the homeostatic mechanism in responding to sleep deprivation, and the phase angle of entrainment. Understanding these will require either identification of mutant genes from model organisms that can be tested in the human population or identification of Mendelian phenotypes of these types that will allow the kind of genetic approaches outlined here for FASPS.

We have now recognized a growing number of autosomal dominant families with FDSPS. Given that DSPS is more common in the general population, it is not yet clear how difficult it will be to identify genetic variants contributing to FDSPS, but efforts are under way to identify such mutations.

\section{REFERENCES}

Archer S.N., Robilliard D.L., Skene D.J., Smits M., Williams A., Arendt J., and von Schantz M. 2003. A length polymorphism in the circadian clock gene Per3 is linked to delayed sleep phase syndrome and extreme diurnal preference. Sleep 26: 413.

Carpen J.D., Archer S.N., Skene D.J., Smits M., and von Schantz M. 2005. A single-nucleotide polymorphism in the $5^{\prime}$-untranslated region of the hPER 2 gene is associated with diurnal preference. J. Sleep Res. 14: 293.

Carpen J.D., von Schantz M., Smits M., Skene D.J., and Archer S.N. 2006. A silent polymorphism in the PER1 gene associates with extreme diurnal preference in humans. J. Hum. Genet. 51: 1122.

Ebisawa T., Uchiyama M., Kajimura N., Mishima K., Kamei Y., Katoh M., Watanabe T., Sekimoto M., Shibui K., Kim K., Kudo Y., Ozeki Y., Sugishita M., Toyoshima R., Inoue Y., Yamada N., Nagase T., Ozaki N., Ohara O., Ishida N., Okawa M., Takahashi K., and Yamauchi T. 2001. Association of structural polymorphisms in the human period3 gene with delayed sleep phase syndrome. EMBO Rep. 2: 342 .

Jones C.R., Campbell S.S., Zone S.E., Cooper F., DeSano A., Murphy P.J., Jones B., Czajkowski L., and Ptáček L.J. 1999. Familial advanced sleep-phase syndrome: A short-period circadian rhythm variant in humans (see comments). Nat. Med. 5: 1062 .

Katzenberg D., Young T., Finn L., Lin L., King D.P., Takahashi J.S., and Mignot E. 1998. A CLOCK polymorphism associated with human diurnal preference. Sleep 21: 569.

Mishima K., Tozawa T., Satoh K., Saitoh H., and Mishima Y. 2005 . The $3111 \mathrm{~T} / \mathrm{C}$ polymorphism of hClock is associated with evening preference and delayed sleep timing in a Japanese population sample. Am. J. Med. Genet. B Neuropsychiatr. Genet. 133: 101

Robilliard D.L., Archer S.N., Arendt J., Lockley S.W., Hack L.M., English J., Leger D., Smits M.G., Williams A., Skene D.J., and Von Schantz M. 2002. The 3111 Clock gene polymorphism is not associated with sleep and circadian rhythmicity in phenotypically characterized human subjects. $J$. Sleep Res. 11: 305.

Roenneberg T., Kuehnle T., Pramstaller P.P., Ricken J., Havel M., Guth A., and Merrow M. 2004. A marker for the end of adolescence. Curr. Biol. 14: R1038.

Takano A., Uchiyama M., Kajimura N., Mishima K., Inoue Y., Kamei Y., Kitajima T., Shibui K., Katoh M., Watanabe T., Hashimotodani Y., Nakajima T., Ozeki Y., Hori T., Yamada N., Toyoshima R., Ozaki N., Okawa M., Nagai K., Takahashi K., Isojima Y., Yamauchi T., and Ebisawa T. 2004. A missense variation in human casein kinase I epsilon gene that induces functional alteration and shows an inverse association with circadian rhythm sleep disorders. Neuropsychopharmacology 29: 1901.

Toh K.L., Jones C.R., He Y., Eide E.J., Hinz W.A., Virshup D.M., Ptáček L.J., and Fu Y.H. 2001. An hPer2 phosphorylation site mutation in familial advanced sleep phase syndrome. Science 


\section{CIRCADIAN RHYTHM GENETICS IN HUMANS}

291: 1040.

Xu Y., Toh K.L., Jones C.R., Shin J.Y., Fu Y.H., and Ptáček L.J. 2007. Modeling of a human circadian mutation yields insights into clock regulation by PER2. Cell 128: 59 .

Xu Y., Padiath Q.S., Shapiro R.E., Jones C.R., Wu S.C., Saigoh N., Saigoh K., Ptáček L.J., and Fu Y.H. 2005. Functional con- sequences of a CKIdelta mutation causing familial advanced sleep phase syndrome. Nature 434: 640 .

Zavada A., Gordijn M.C., Beersma D.G., Daan S., and Roenneberg T. 2005. Comparison of the Munich Chronotype Questionnaire with the Horne-Ostberg's MorningnessEveningness score. Chronobiol. Int. 22: 267. 


\title{
$\overbrace{\text { CSH' }}^{\infty}$ Cold Spring Harbor Symposia SYMPOSIA on Quantitative Biology
}

\section{Novel Insights from Genetic and Molecular Characterization of the Human Clock}

\author{
L. J. Ptácek, C. R. Jones and Y.-H. Fu
}

Cold Spring Harb Symp Quant Biol 2007 72: 273-277

Access the most recent version at doi:10.1101/sqb.2007.72.017

References This article cites 14 articles, 2 of which can be accessed free at:

http://symposium.cshlp.org/content/72/273.full.html\#ref-list-1

License

Email Alerting Receive free email alerts when new articles cite this article - sign up in the box at the Service top right corner of the article or click here. 